Discrete Comput Geom 26:499-511 (2001)

DOI: $10.1007 / \mathrm{s} 00454-001-0045-8$

\title{
Approximation for Minimum Triangulations of Simplicial Convex 3-Polytopes*
}

\author{
F. Y. L. Chin, ${ }^{1}$ S. P. Y. Fung, ${ }^{1}$ and C.-A. Wang ${ }^{2}$ \\ ${ }^{1}$ Department of Computer Science and Information Systems, \\ The University of Hong Kong, \\ Pokfulam Road, Hong Kong \\ \{chin,pyfung\}@csis.hku.hk \\ ${ }^{2}$ Department of Computer Science, Memorial University of Newfoundland, \\ St. John's, Newfoundland, Canada A1B 3X5 \\ wang@cs.mun.ca
}

\begin{abstract}
A minimum triangulation of a convex 3-polytope is a triangulation that contains the minimum number of tetrahedra over all its possible triangulations. Since finding minimum triangulations of convex 3-polytopes was recently shown to be NP-hard, it becomes significant to find algorithms that give good approximation. In this paper we give a new triangulation algorithm with an improved approximation ratio $2-\Omega(1 / \sqrt{n})$, where $n$ is the number of vertices of the polytope. We further show that this is the best possible for algorithms that only consider the combinatorial structure of the polytopes.
\end{abstract}

\section{Introduction}

Triangulation is the subdivision of $d$-dimensional polytopes into simplices, such that these simplices only intersect at common (possibly empty) faces. Triangulation has important applications in computer-aided design, computer graphics, finite element analysis, etc. In this paper we are concerned with triangulations of three-dimensional convex polytopes without introducing Steiner points, i.e. all vertices of the simplices must be from the original polytope.

Triangulation in three dimensions (also called tetrahedralization) is to partition the polytope into a set of tetrahedra. Three-dimensional triangulations have many interesting properties. Convex polytopes can always be triangulated, but non-convex polytopes may

\footnotetext{
* The work by the first author was supported by RGC Grant HKU 7019/00E. The third author's work was supported by NSERC Grant OPG0041629.
} 
not: the Schönhardt polytope [Sc] is such an example. It is even NP-complete to determine whether a given non-convex polytope can be triangulated (without using Steiner points) [RS2]. Different triangulations of the same convex polytope may result in different sizes, i.e. having different numbers of tetrahedra [BE]. Minimum triangulations of a polytope are triangulations that have the minimum size. Note that minimum triangulations may not be unique for a polytope, i.e. there may be several triangulations having the same minimum size, e.g. an octahedron. The complexity of finding a minimum triangulation was a major open problem [BE], and only quite recently it was shown to be NP-hard [BDR1], [BDR2]. This raises the question of finding good approximation algorithms for this problem.

We first state a few terms and definitions. We consider polytopes with vertices in general position, i.e. no four vertices are coplanar. This implies that the polytope is simplicial. A cap of a vertex $v$ in a polytope $P$ is the region between $P$ and the convex hull $(\mathrm{CH})$ of $P \backslash\{v\}$. An edge in a triangulation is called an interior edge if it does not lie on the surface of the polytope. A 3-cycle is a closed path of three edges on the surface graph of a polytope such that each side of the 3-cycle contains at least one other vertex. Throughout this paper let $n$ denote the number of vertices of the polytope $P$, let $e_{m}$ be the number of interior edges in a minimum triangulation of $P$, and let $\Delta$ be the maximum vertex degree on the surface graph of $P$. For any polytope, the number of interior edges $e$ in a triangulation is directly related to the number of tetrahedra $t$ by the formula $t=e+n-3$ [BBDR]. This implies a lower bound of $n-3$ tetrahedra for any triangulation.

There are two common ways to triangulate a convex polytope $P$. One way is to remove the cap of a vertex $v$ (which can easily be triangulated) to get a smaller polytope $C H(P \backslash\{v\})$, and then to iterate this process for $C H(P \backslash\{v\})$. This is known as "peeling" [EPW]. Peeling a vertex $v$ of degree $\operatorname{deg}(v)$ gives $\operatorname{deg}(v)-2$ tetrahedra. Using the "greedy peeling" heuristic, it can be shown to yield an approximation ratio of $3-\Omega(1 / n)$. "Pulling" [Le] (also called "starring" [Be]) is another well-known approach. Pulling picks a highest-degree vertex of the polytope and uses it to form tetrahedra with all faces not containing $v$, producing $2 n-4-\Delta \leq 2 n-7$ tetrahedra, which, considering the $n-3$ lower bound, gives an approximation ratio of $2-\Omega(1 / n)$.

The $n-3$ lower bound is tight, as achieved by "stacked" polytopes [RS1], [BDR2]. These polytopes contain many 3 -cycles. This suggests that partitioning a polytope along 3-cycles (if any) may yield a smaller triangulation. However, it is shown in [STT] that there exist polytopes requiring as many as $2 n-10$ tetrahedra for any triangulation. For $n>12$, we have $\Delta \geq 6$, and the pulling heuristic gives no more than $2 n-10$ tetrahedra. Thus the pulling heuristic is worst-case optimal, in terms of the absolute number of tetrahedra produced. In terms of the approximation ratio, however, we can use the idea of 3 -cycles to give a better heuristic with ratio $2-\Omega(1 / \sqrt{n})$.

Despite the small improvement, we show that no other approximation algorithm can give a better ratio, under the restriction that only the combinatorial structure of the polytope is considered. By the combinatorial structure we mean the inclusion relation between the faces, as represented by the face lattice [Zi]. Two polytopes are combinatorially equivalent (i.e. have the same combinatorial structure) if there is a bijection between their faces that preserves the inclusion relation. It is known that two 3-polytopes are combinatorially equivalent if and only if their surface graphs are isomorphic. Two polytopes 
that have the same combinatorial structure may have different coordinatizations, i.e. having different vertex coordinates. In their paper [BBDR], Below et al. showed that the size of minimum triangulations of a polytope is not an invariant of the polytope's face lattice, i.e. polytopes with the same face lattice may have different minimum-size triangulations. They arrived at this conclusion by considering something called the "vertex-edge chain structure," and showed that it has the property whereby the size of triangulation of a polytope with that structure changes greatly depending on the presence of a single interior edge. We extend their result to show that this difference can indeed be very large, and use this to prove a lower bound of $2-O(1 / \sqrt{n})$ on the approximability of this problem. This shows that our algorithm is the best possible in this sense.

The paper is organized as follows. In Section 2 we first prove some extended properties of the vertex-edge chain structures. Then in Section 3 we modify the pulling heuristic to obtain a better bound of $2-\Omega(1 / \sqrt{n})$ on the approximation ratio. Finally, in Section 4 we show that any algorithm that only considers the combinatorial structure of a polytope cannot give an approximation ratio better than $2-O(1 / \sqrt{n})$. We conclude with some open problems.

\section{Vertex-Edge Chain Structure}

The so-called "vertex-edge chain structure" (VECS) that appeared in [BBDR] is used extensively in this paper. It consists of $2 m+2$ faces $\left(a, q_{i}, q_{i+1}\right)$ and $\left(b, q_{i}, q_{i+1}\right)$ for $i=0, \ldots, m$, with the additional restriction that the line segment $q_{0} q_{m+1}$ goes through the interior of the polytope $C H\left(a, b, q_{0}, q_{1}, \ldots, q_{m+1}\right)$; we call this polytope a wedge (Fig. 1). All the $2 m+2$ faces form a convex surface. We say this VECS has size $m$, and call edge $a b$ the main diagonal. A tetrahedron in a triangulation is incident to a VECS if at least three out of its four vertices lie on the VECS.

A VECS of size $m$ can be triangulated using $m+1$ incident tetrahedra $a b q_{i} q_{i+1}$ $(0 \leq i \leq m)$ by one interior edge $a b$. However, if the main diagonal is not allowed to appear in the triangulation, the situation is different: in [BBDR] it was proved that if $a b$ is not in the triangulation, then at least $m$ other interior edges must be present, and this implies a large number of incident tetrahedra. We now extend this and state some slightly stronger results concerning VECS.

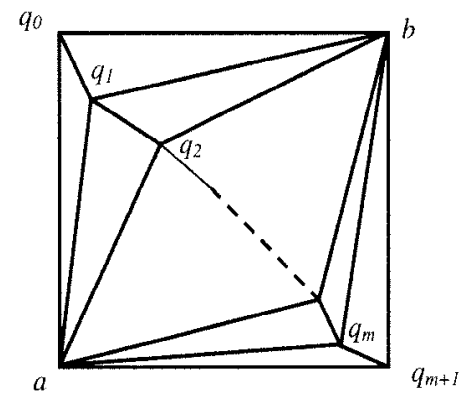

Fig. 1. A VECS of size $m$. 


\section{Lemma 1.}

(i) In a polytope containing a VECS of size $m$ as a substructure, if the main diagonal is not present in a triangulation, at least $2 m$ tetrahedra must be incident to the VECS

(ii) If a polytope contains $k$ mutually disjoint VECSs, each of size m, as substructures, and all without the main diagonals in a triangulation, then in this triangulation there exist at least $2 m k$ tetrahedra incident to these VECSs.

Proof. (i) There are $2 m+2$ faces in the VECS. If each face is associated with a separate tetrahedron, then there exist at least $2 m$ tetrahedra. Faces $a q_{i} q_{i+1}$ and $b q_{i} q_{i+1}$ cannot be in the same tetrahedron because this will induce edge $a b$. If $a q_{i-1} q_{i}$ and $a q_{i} q_{i+1}$ are in the same tetrahedron (similarly for $b q_{i-1} q_{i}$ and $b q_{i} q_{i+1}$ ), $a q_{i-1} q_{i+1}$ will be a new face incident to this VECS, which determines another incident tetrahedron. If this new face is in the same tetrahedron as another face, this will in turn determine another incident tetrahedron. This process can continue until the two faces $a q_{0} q_{m+1}$ and $b q_{0} q_{m+1}$ appear. A simple induction argument on this idea can show that at least $2 m$ tetrahedra are incident to this VECS.

(ii) A tetrahedron incident to one of the VECSs has at least three vertices on that VECS, leaving at most one vertex, thus this tetrahedron cannot be incident to other VECSs. It follows from (i) that the $k$ VECSs must have $2 m k$ distinct incident tetrahedra.

Two VECSs $\left(a, b, q_{0}, \ldots, q_{m+1}\right)$ and $\left(a^{\prime}, b^{\prime}, q_{0}^{\prime}, \ldots, q_{m^{\prime}+1}^{\prime}\right)$ are interlocked if $a b$ intersects faces $a^{\prime} b^{\prime} q_{0}^{\prime}$ and $a^{\prime} b^{\prime} q_{m^{\prime}+1}^{\prime}$, and $a^{\prime} b^{\prime}$ intersects faces $a b q_{0}$ and $a b q_{m+1}$. More generally, $k>2$ VECSs are pairwise-interlocked (or simply "interlocked") if any two of them are interlocked.

Lemma 2. In any triangulation of a polytope containing $k$ pairwise-interlocked VECSs, each of size $m$, there exists at most one VECS having fewer than $2 m$ incident tetrahedra.

Proof. If a VECS has fewer than $2 m$ incident tetrahedra, its main diagonal must be used in the triangulation: two triangular faces of that VECS have to form a tetrahedron with the main diagonal (see proof of Lemma 1). The main diagonals of any other interlocked VECSs, if used, will penetrate this tetrahedron, thus cannot be in the triangulation. Therefore all other VECSs cannot have fewer than $2 m$ incident tetrahedra.

\section{The Triangulation Algorithm}

\subsection{A Restricted Case}

We first consider the case where the given polytope has no 3-cycles. This restriction will be removed in the next subsection. We analyze the approximation ratio of the pulling heuristic in this case. 
Lemma 3. Let $P$ be a convex polytope that has no 3-cycles, $n>4$. Then:

(i) At least one interior edge must be incident to the cap of any vertex.

(ii) $e_{m}+\Delta \geq \sqrt{2 n}-1$.

Proof. (i) Consider a particular vertex $v_{0}$ and its neighbors $v_{1}, v_{2}, \ldots, v_{k}$ where $4 \leq$ $k \leq \Delta$ (note that if $k=3, P$ contains a 3 -cycle). The triangle $v_{0} v_{1} v_{2}$ must be associated with a tetrahedron. If the fourth vertex $v$ of this tetrahedron is one from $v_{3}, v_{4}, \ldots, v_{k}$, then either $v_{1} v$ or $v_{2} v$ (or both) is an interior edge (otherwise $v_{0} v_{1} v$ or $v_{0} v_{2} v$ would be a 3-cycle). If $v$ is not a vertex from $v_{3}, v_{4}, \ldots, v_{k}$, then it is not a neighbor of $v_{0}$ and hence $v_{0} v$ is an interior edge. In either case, at least one interior edge is incident to the cap of $v_{0}$.

(ii) Suppose we count the number of interior edges by counting the number of interior edges incident to all the $n$ possible caps. By (i) above, this number is at least $n$. However, each interior edge is counted more than once; it is counted by the caps of their two endpoints as well as the endpoints' neighbors. The total number of these caps does not exceed $2(\Delta+1)$. Therefore each edge is counted at most $2(\Delta+1)$ times, giving $e_{m} \times 2(\Delta+1) \geq n$. Applying the Arithmetic-Geometric-Mean inequality, $\left(e_{m}+(\Delta+\right.$ 1)) $/ 2 \geq \sqrt{e_{m}(\Delta+1)}$, giving $e_{m}+\Delta \geq \sqrt{2 n}-1$.

The approximation ratio of the pulling heuristic under the no-3-cycle restriction is shown in the lemma below.

Lemma 4. Using the pulling heuristic, the approximation ratio $r$ is bounded above by $(2 n-\sqrt{2 n}-2) /(n-2)$.

Proof. The pulling heuristic produces $2 n-4-\Delta$ tetrahedra while the size of minimum triangulations is $e_{m}+n-3$. Thus the approximation ratio is bounded by $(2 n-4-$ $\Delta) /\left(n-3+e_{m}\right)$. Hence, by Lemma 3(ii),

$$
r \leq \frac{2 n-4-\left(\sqrt{2 n}-1-e_{m}\right)}{n-3+e_{m}}=\frac{2 n-\sqrt{2 n}-2+\left(e_{m}-1\right)}{n-2+\left(e_{m}-1\right)} \leq \frac{2 n-\sqrt{2 n}-2}{n-2},
$$

since $e_{m} \geq 1$ by Lemma 3(i).

\subsection{The General Case}

We now deal with the general case for polytopes having 3-cycles. Our algorithm is very simple:

- Step 1. Find all the 3-cycles, using algorithms such as those in [PY] and [CN].

- Step 2. Partition the polytope along all these 3-cycles to produce a set of subpolytopes, each is free of 3-cycles.

- Step 3. Apply the pulling heuristic to each resulting subpolytope.

We first consider the time complexity of this algorithm. Finding all 3-cycles takes linear time [PY], [CN]. Step 2 can be done as follows: Each 3-cycle divides the surface graph 
into an interior part and an exterior part. Starting from any 3-cycle, we visit all vertices inside this 3-cycle; whenever we encounter edges on other 3-cycles nested in the current 3-cycle, we process this nested 3-cycle first. In this depth-first search manner, we first produce the deepest nested subpolytope. We remove this subpolytope and continue to visit the vertices of the current subpolytope, until we are finished with this 3-cycle. We process similarly for the exterior part until all 3-cycles are processed. All the search and removal can be done in time proportional to the size of the components, thus they take $O(n)$ time in total. Finally, picking vertices of maximum degree and pulling for all subpolytopes also take $O(n)$ time in total. Therefore we have

Fact 1. The algorithm takes $\Theta(n)$ time.

In the following we prove that for polytopes with 3-cycles the algorithm achieves the same approximation ratio as the special case in the previous subsection.

Lemma 5. Let $P$ be a convex polytope with maximum degree $\Delta$ and has a 3-cycle. Suppose we cut through the 3-cycle to produce two polytopes $P_{1}$ and $P_{2}$, having maximum degrees $\Delta_{1}$ and $\Delta_{2}$, respectively. Then $\Delta \leq \Delta_{1}+\Delta_{2}-2$.

Proof. Clearly, $3 \leq \Delta_{1} \leq \Delta, 3 \leq \Delta_{2} \leq \Delta$. We apply a case-by-case analysis.

Case 1 . Some vertices with degree $\Delta$ are on one side of $P_{1}$ and $P_{2}$, but not on the 3-cycle, say $\Delta_{1}=\Delta$. Then $\Delta_{1}+\Delta_{2}-2 \geq \Delta+3-2>\Delta$.

Case 2. All vertices with degree $\Delta$ are on the 3-cycle being cut. Let the degrees of the two new vertices cut from the vertex with degree $\Delta$ be $d_{1}$ and $d_{2}$, respectively. Clearly, $\Delta=d_{1}+d_{2}-2$. Since $\Delta_{1} \geq d_{1}, \Delta_{2} \geq d_{2}$, we have $\Delta \leq \Delta_{1}+\Delta_{2}-2$.

Thus in all cases the inequality holds.

Lemma 6. Let $P, P_{1}$ and $P_{2}$ be the same polytopes as defined in Lemma 5, with $n, n_{1}$ and $n_{2}$ vertices, respectively. Let $t, t_{1}$ and $t_{2}$ be the number of tetrahedra produced by the pulling heuristic applied to $P, P_{1}$ and $P_{2}$, respectively. Then $t \geq t_{1}+t_{2}$.

Proof. Note that $t=2 n-4-\Delta, t_{1}=2 n_{1}-4-\Delta_{1}$, and $t_{2}=2 n_{2}-4-\Delta_{2}$. Thus

$$
\begin{aligned}
t_{1}+t_{2} & =2\left(n_{1}+n_{2}\right)-8-\left(\Delta_{1}+\Delta_{2}\right)=2(n+3)-8-\left(\Delta_{1}+\Delta_{2}\right) \\
& =2 n-4-\left(\Delta_{1}+\Delta_{2}-2\right) \leq 2 n-4-\Delta=t .
\end{aligned}
$$

The last inequality follows from Lemma 5.

The above lemma shows that cutting along a 3-cycle and separately pulling on each piece will not produce more tetrahedra than that of pulling on the original polytope. Note also that any cut will not create new 3-cycles since no new surface edge is created, and two 3-cycles will never "cross" each other. Therefore the lemma is also true for multiple cuts. 
Lemma 7. For a polytope with $k 3$-cycles, the algorithm produces a triangulation with at most $2 n-7-k$ tetrahedra.

Proof. We prove this by induction on $k$. When $k=0$, the algorithm reduces to a single pulling, which gives $2 n-4-\Delta \leq 2 n-7$ tetrahedra, so the claim holds. For $k>0$, assume the statement is true for all $k^{\prime}<k$. For a polytope with $k 3$ cycles, the algorithm picks a 3 -cycle and partitions the polytope into two subpolytopes. Suppose they have $n_{1}$ and $n_{2}$ vertices, and $k_{1}$ and $k_{2} 3$-cycles, respectively. We have $n=n_{1}+n_{2}-3,0 \leq k_{1}<k, 0 \leq k_{2}<k, k=k_{1}+k_{2}+1$. Since each subpolytope has fewer than $k 3$-cycles, by induction assumption their triangulations have size at most $2 n_{1}-7-k_{1}$ and $2 n_{2}-7-k_{2}$, respectively. So the size of triangulation of the polytope is at most $\left(2 n_{1}-7-k_{1}\right)+\left(2 n_{2}-7-k_{2}\right)=2(n+3)-14-\left(k_{1}+k_{2}\right)=$ $2 n-7-\left(k_{1}+k_{2}+1\right)=2 n-7-k$. Thus the claim holds.

Theorem 1. The algorithm gives a bound of $2-\Omega(1 / \sqrt{n})$ on the approximation ratio.

Proof. Suppose there are $k 3$-cycles in $P$, and we apply $k$ cuts to partition $P$ into $k+1$ parts, each free of 3-cycles. Note that $k=O(n)$. Consider the following two cases:

Case 1: $k=o(n)$. Since each 3-cycle contains exactly three vertices, there are at most $3 k$ vertices lying on 3-cycles. There are at most $k$ degree-3 vertices, since each of them must determine a 3 -cycle. Thus there are at least $n-4 k=\Theta(n)$ vertices that are neither lying on 3-cycles nor of degree-3. By the same argument as in Lemma 3(i), each of these $\Theta(n)$ vertices has an interior edge incident to its corresponding cap in any triangulation. Like Lemma 3(ii), we have $2 e_{m}(\Delta+1) \geq \Theta(n)$, giving $e_{m}+\Delta \geq \Theta(\sqrt{n})$. Using a proof similar to that of Lemma 4 , we have $r \leq(2 n-4-\Delta) /\left(e_{m}+n-3\right) \leq 2-\Omega(1 / \sqrt{n})$. This means even if we apply the pulling heuristic directly to the original polytope (without cutting 3-cycles), we still have a bound of $2-\Omega(1 / \sqrt{n})$. By Lemma 6 , partitioning the polytope along all 3-cycles before pulling will not increase the number of tetrahedra. Thus our algorithm gives a bound of $2-\Omega(1 / \sqrt{n})$ in this case.

Case 2: $k=\Theta(n)$. By Lemma 7, the approximation ratio is thus

$$
r \leq \frac{2 n-7-c n}{e_{m}+n-3} \leq 2-c<2-\Omega\left(\frac{1}{\sqrt{n}}\right)
$$

for some constant $c>0$.

Although cutting 3-cycles is an important step in our algorithm, it does not always produce minimum triangulations. A counterexample can be constructed using a "cupola" [BDR2], i.e. three VECSs of size $m$ attached to the three non-convex sides of a Schönhardt polytope. We add a vertex joining to the triangular bottom face of the cupola, making sure that it is inside the "visibility cone" of the top face, and the whole polytope remains convex (Fig. 2). Now the original bottom face becomes a 3-cycle. This polytope has $3 m+7$ vertices. If we cut through the 3-cycle, the three main diagonals cannot be used together, because then the Schönhardt polytope cannot be triangulated. Hence at least one main diagonal cannot be used, and therefore the cupola needs at least $4 m$ tetrahedra 


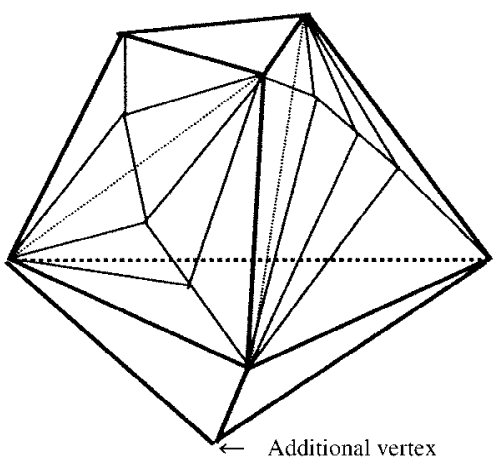

Fig. 2. A cupola with a 3-cycle.

to triangulate. On the other hand, since the new vertex can see all non-convex faces of the Schönhardt polytope from inside, we can triangulate the Schönhardt polytope by pulling from the added vertex, while the three VECSs are triangulated using their own main diagonals. This gives only $3 m+10$ tetrahedra, showing that cutting 3-cycles does not always produce minimum triangulations.

Note that finding 3-cycles, cutting them, and pulling the resulting subpolytopes, all these depend only on the combinatorial structure of the polytope. In other words, they only require the surface graph of the polytope to be given. We show in the next section that this restriction implies a lower bound of approximability.

\section{Lower Bound of Approximation}

In this section we extend the idea in [BBDR] to show a large difference in the size of minimum triangulations for two different convex polytopes with the same face lattice. As a major result we show that, given only the face lattice, no approximation algorithms for finding minimum triangulations of convex polytopes can have an approximation ratio better than $2-O(1 / \sqrt{n})$.

The idea for our proof is to construct two combinatorially equivalent polytopes, P1 and $\mathrm{P} 2$, such that their minimum triangulations differ by a large number of tetrahedra. Both polytopes consist of $m$ thin wedges, each wedge being a VECS of size $m$. The polytopes have the same number of vertices $n$ where $n=m^{2}+4 m$. In P1 the $m$ VECSs are pairwise-interlocked, thus only one of the $m$ main diagonals can be present in any triangulation, resulting in a greater number of tetrahedra. P2 is constructed similarly but the wedges are not interlocked, and thus all $m$ main diagonals can be present in a triangulation, resulting in a much smaller number of tetrahedra.

\subsection{The Detailed Construction}

Figure 3 shows a rough idea of the construction. We first arrange $m$ wedges in an $x y z$ coordinate system. Wedge $W_{k}(1 \leq k \leq m)$ has vertices $a_{k}, b_{k}, c_{k}, d_{k}$ where $a_{k} b_{k}$ is the 


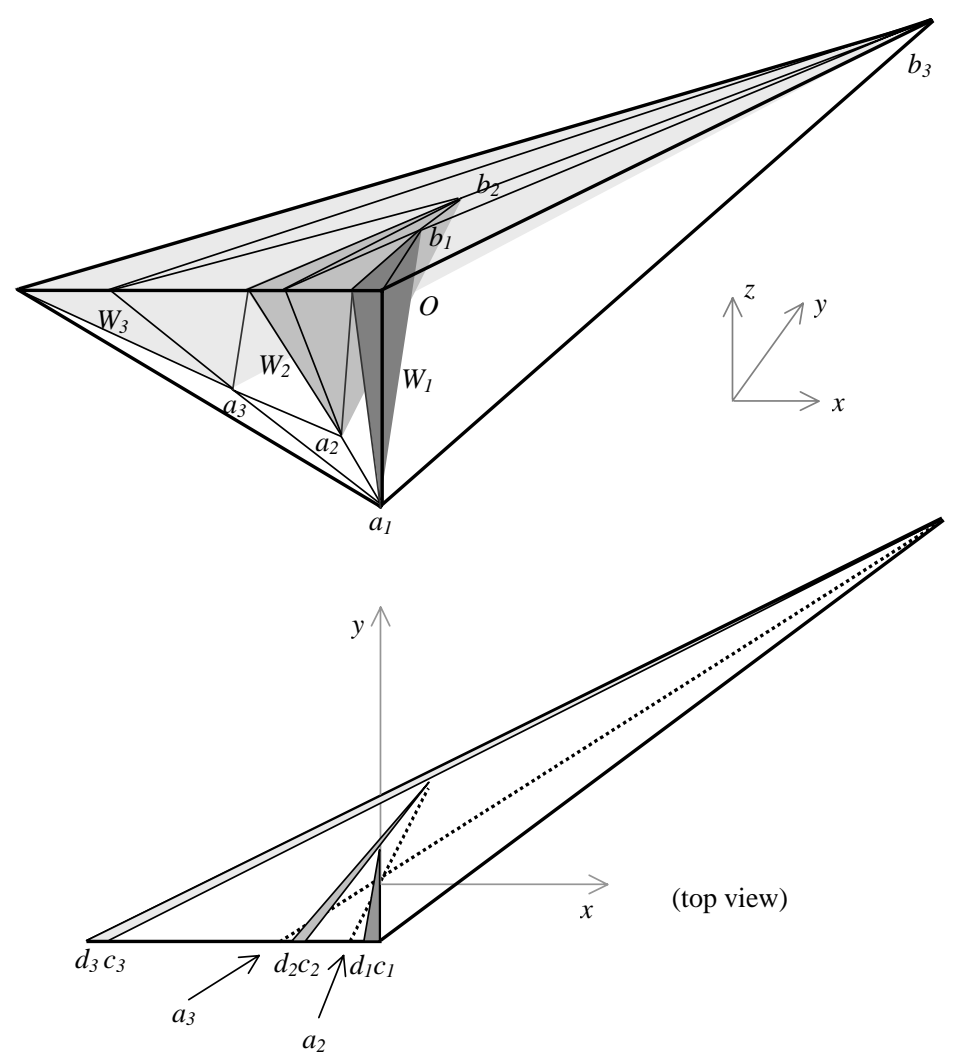

Fig. 3. Construction of $\mathrm{P} 1$ and $\mathrm{P} 2$, showing three wedges. $q^{i}$,s are not shown

main diagonal. All faces $a_{k} c_{k} d_{k}$ lie on the vertical plane $y=-1$ and all faces $b_{k} c_{k} d_{k}$ lie on the horizontal plane $z=1 . W_{1}$ is at $a_{1}(0,-1,-1), b_{1}(0,1,1), c_{1}(0,-1,1)$, $d_{1}(-0.1,-1,1)$. Assuming wedges $W_{1}$ to $W_{k}$ have been constructed, $W_{k+1}$ is constructed as follows (Fig. 3 also shows a top view from the positive $\mathrm{z}$-axis):

Pick $a_{k+1}$ so that its $x$-coordinate is smaller than $d_{k}$ and its $z$-coordinate is larger than $a_{k}$, and such that $a_{1}, a_{2}, \ldots, a_{k}, a_{k+1}$ form a convex chain with respect to the point ( 0 , $-1,-\infty) . b_{k+1}$ is uniquely determined on the $z=1$ plane such that $a_{k+1} b_{k+1}$ passes through the origin $(0,0,0)$. Find a point $c_{k+1}$ such that $b_{k+1} c_{k+1}$ does not intersect all previous wedge faces on the horizontal plane. $d_{k+1}$ is placed slightly to the "left" (negative $x$ direction) of $c_{k+1}$.

The following two facts are not difficult to establish:

1. $b_{k+1}$ has larger $y$-coordinate than $b_{k}$.

2. Vertices $b_{1}, b_{2}, \ldots, b_{k}, b_{k+1}$ form a convex chain with respect to the point $(\infty$, $0,1)$.

Moreover, $m$ points $q_{k}^{1}, q_{k}^{2}, \ldots, q_{k}^{m}$ are created between each interval $\left(c_{k}, d_{k}\right)$ and edges $a_{k} q_{k}^{i}, b_{k} q_{k}^{i}(1 \leq i \leq m)$ are added so that they form $m$ VECSs each of size $m$. 
This construction puts all the vertices on two planes. This degeneracy can be removed by slightly bending the horizontal and vertical planes so that the polytope becomes strictly convex, while keeping all the main diagonals intersecting at the origin.

Now all the wedges intersect at a single point $(0,0,0)$. For P1, we apply a final step, moving all the wedges slightly along the positive $x$-axis: the $k$ th wedge $W_{k}$ is moved by a distance of $k \delta$ where $\delta>0$ is a small constant. This makes any two wedges interlocked. The surface graph of the polytope will not be changed if $\delta$ is sufficiently small. P1 is formed by taking the convex hull of all the wedges, having the following edges:

$$
\begin{aligned}
& a_{k} c_{k}, a_{k} d_{k}, b_{k} c_{k}, b_{k} d_{k}(1 \leq k \leq m) ; \\
& d_{k} a_{k+1}, d_{k} c_{k+1}, b_{k} c_{k+1}, a_{k} a_{k+1}, b_{k} b_{k+1}(1 \leq k \leq m-1) ; \\
& a_{1} a_{k}, b_{1} b_{k}(3 \leq k \leq m) ; \\
& q_{k}^{i} q_{k}^{i+1}, q_{k}^{1} c_{k}, q_{k}^{m} d_{k}(1 \leq i \leq m-1,1 \leq k \leq m) ; \\
& q_{k}^{i} a_{k}, q_{k}^{i} b_{k}(1 \leq i \leq m, 1 \leq k \leq m) ; \\
& c_{1} b_{m}, a_{1} b_{m}, a_{1} d_{m} .
\end{aligned}
$$

The construction of P2 is almost identical to that of P1, except in the final step the wedges do not move but shrink: we draw a vertical line passing through each vertex $c_{k}$ and intersect edge $d_{k} a_{k}$ at $a_{k}^{\prime}$. (By construction, $c_{k}$ must be on the "left" of $a_{k}$.) We shrink wedge $W_{k}$ from $a_{k} b_{k} c_{k} d_{k}$ to $a_{k}^{\prime} b_{k} c_{k} d_{k}$. Then all wedges do not intersect, as can be seen in Fig. 3 where $a_{k}$ coincides with $c_{k}$ in the top view after shrinking. The chain $a_{1}^{\prime}\left(=a_{1}\right), a_{2}^{\prime}, \ldots, a_{m}^{\prime}$ is still convex, and the surface graph will not be changed by this transformation. Thus we have the following:

Claim 1. $\mathrm{P} 1$ and $\mathrm{P} 2$ have the following properties:

(i) Both $\mathrm{P} 1$ and $\mathrm{P} 2$ are strictly convex.

(ii) $\mathrm{P} 1$ and $\mathrm{P} 2$ have the same surface graph.

(iii) All VECS faces $a_{k} q_{k}^{i} q_{k}^{i+1}$ and $b_{k} q_{k}^{i} q_{k}^{i+1}$ are on the surface of $\mathrm{P} 1$ or $\mathrm{P} 2$.

(iv) For $\mathrm{P} 1$, all wedges are interlocked. For $\mathrm{P} 2$, all wedges do not intersect one another.

\subsection{Size of the Triangulations}

We next show that minimum triangulations of P1 contain a much greater number of tetrahedra than those of $\mathrm{P} 2$.

Lemma 8. Any minimum triangulation of $\mathrm{P} 1$ contains at least $2 m^{2}-m+1$ tetrahedra.

Proof. By Lemmas 1 and 2, among the $m$ main diagonals of the interlocked wedges, only one can be present in any triangulation of P1. Note that all $m$ VECSs are disjoint in our construction, therefore at least $(m-1)(2 m)$ tetrahedra must be incident to these $m-1$ VECSs not using their main diagonals. The VECS with the main diagonal creates $m+1$ tetrahedra. Thus, a total of at least $(m-1)(2 m)+(m+1)=2 m^{2}-m+1$ tetrahedra must appear in any triangulation. 


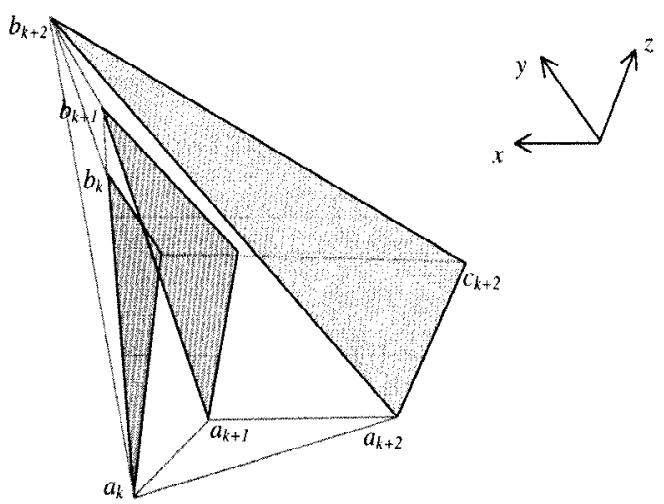

Fig. 4. The space between three wedges, viewing from "the back". The thickness of each wedge is not shown.

Lemma 9. Any minimum triangulation of $\mathrm{P} 2$ contains at most $m^{2}+8 m-8$ tetrahedra.

Proof. We triangulate each wedge by using the main diagonal of its corresponding VECS, producing $m+1$ tetrahedra each. Now we have to triangulate the remaining space. Consider any three consecutive wedges $W_{k}, W_{k+1}, W_{k+2}(1 \leq k \leq m-2)$. We add the interior edges $a_{k} b_{k+1}$ and $a_{k+1} b_{k+2}$ so that the two regions $a_{k} b_{k} d_{k} a_{k+1} b_{k+1} c_{k+1}$ and $a_{k+1} b_{k+1} d_{k+1} a_{k+2} b_{k+2} c_{k+2}$ are convex, and they can be triangulated using four tetrahedra each. Finally, the remaining space can be triangulated with three tetrahedra, $a_{k} a_{k+1} a_{k+2} b_{k+2}, b_{k} b_{k+1} b_{k+2} a_{k}$, and $a_{k+1} b_{k+1} a_{k} b_{k+2}$, by adding the edge $a_{k} b_{k+2}$ (Fig. 4). Using the above procedure, wedge $W_{k+1}$ is effectively "shielded" and the triangulation can proceed with the remaining $m-1$ wedges as if there is no $W_{k+1}$. We use this to triangulate P2 starting from $W_{1} W_{2} W_{3}$, then $W_{1} W_{3} W_{4}$, and so on until only two wedges $W_{1}$ and $W_{m}$ remain. The space beyond $W_{1}$ and $W_{m}$ can be triangulated by adding the tetrahedra $a_{1} b_{1} c_{1} b_{m}$ and $a_{m} b_{m} d_{m} a_{1}$ for the two outer faces. This completes the triangulation.

There are $m$ VECSs, each giving $m+1$ tetrahedra, each of the $m-1$ convex regions between $W_{k}$ and $W_{k+1}$ gives four tetrahedra, each of the $m-2$ inductive steps gives three more tetrahedra, and the final step gives two more. Thus there is a triangulation with total number of tetrahedra $m(m+1)+4(m-1)+3(m-2)+2=m^{2}+8 m$ -8 .

Theorem 2. Any approximation algorithm for finding minimum triangulations of convex polytopes, provided with only the face lattice, cannot have an approximation ratio better than $2-O(1 / \sqrt{n})$.

Proof. By Lemmas 8 and 9, a minimum triangulation of P1 has at least $2 m^{2}-m+1=$ $2 n-\Theta(\sqrt{n})$ tetrahedra while that of P2 has at most $m^{2}+8 m-8=n+\Theta(\sqrt{n})$ tetrahedra. Note that P1 and P2 are combinatorially equivalent but have different coordinates. Since minimum triangulations of two combinatorially equivalent polytopes can have either at least $2 n-\Theta(\sqrt{n})$ or at most $n+\Theta(\sqrt{n})$ tetrahedra, any approxima- 
tion algorithm that considers the face lattice only must produce a triangulation with the worst-case number, i.e. $2 n-\Theta(\sqrt{n})$. Otherwise, this algorithm would produce a triangulation with less than the optimal number of tetrahedra in some cases, which would be a contradiction. Since a minimum triangulation may in fact contain at most $n+\Theta(\sqrt{n})$ tetrahedra, the approximation ratio is bounded by $r \geq(2 n-\Theta(\sqrt{n})) /(n+\Theta(\sqrt{n})) \geq$ $2-O(1 / \sqrt{n})$.

\section{Conclusion}

In this paper we give an approximation algorithm for finding a minimum triangulation of convex polytopes with approximation ratio $2-\Omega(1 / \sqrt{n})$. We also show a $2-O(1 / \sqrt{n})$ bound on the approximability of this minimum triangulation problem using combinatorial algorithms. Thus there is no better constant-ratio approximation algorithm without considering the actual coordinates of the vertices. A problem that follows naturally is whether algorithms with better approximation ratios can be achieved, or similar nonapproximability results exist, if vertex coordinates are taken into account. Another interesting problem is the case when the maximum degree is bounded by a constant. It is not known whether the problem remains NP-hard in this case, and it is also unclear whether the bounds we gave can be improved.

Our algorithm is only designed for simplicial polytopes. The non-simplicial case is quite interesting but it seems that there is no easy way to extend our algorithm for such a case with the same approximation ratio. It is not clear if there is a polynomial-time algorithm for this.

\section{References}

[BBDR] Below, A., Brehm, U., De Loera, J., and Richter-Gebert, J., Minimal Simplicial Dissections and Triangulations of Convex 3-Polytopes, Discrete and Computational Geometry 24 (2000), 3548.

[BDR1] Below, A., De Loera, J., and Richter-Gebert, J., Finding Minimal Triangulations of Convex 3Polytopes is NP-Hard, Proceedings of 11th Annual ACM-SIAM Symposium On Discrete Algorithms (2000), pp. 65-66.

[BDR2] Below, A., De Loera, J., and Richter-Gebert, J., The Complexity of Finding Small Triangulations of Convex 3-Polytopes, manuscript, 2000. Available at http://www.math.ucdavis.edu/ deloera/ RECENT_WORK/recent.html.

[Be] Bern, M., Compatible Tetrahedralizations, Proceedings of 9th ACM Symposium on Computational Geometry (1993), pp. 281-288.

[BE] Bern, M., and Eppstein, D., Mesh Generation and Optimal Triangulation, in Computing in Euclidean Geometry, World Scientific, Singapore, 1995.

[CN] Chiba, N., and Nishizeki, T., Arboricity and Subgraph Listing Algorithms, SIAM Journal on Computing 14 (1985), 210-223.

[EPW] Edelsbrunner, H., Preparata, F. P., and West, D. B., Tetrahedrizing Point Sets in Three Dimensions, Journal of Symbolic Computation 10 (1990), 335-347.

[Le] Lee, C. W., Subdivision and Triangulations of Polytopes, in Handbook of Discrete and Computational Geometry (Goodman, J. E., and O’Rourke, J., eds.), CRC Press, Boca Raton, FL, 1997, pp. 271-290.

[PY] Papadimitriou, C. H., and Yannakakis, M., The Clique Problem for Planar Graphs, Information Processing Letters 13 (1981), 131-133. 
[RS1] Rothschild, G. L., and Straus, E. G., On Triangulations of the Convex Hull of $n$ Points, Combinatorica 5 (1985), 167-179.

[RS2] Ruppert, J., and Seidel, R., On the Difficulty of Tetrahedralizing Three-Dimensional Non-convex Polyhedra, Discrete and Computational Geometry 7 (1992), 227-253.

[Sc] Schönhardt, E., Über die Zerlegung von Dreieckspolyedern in Tetraeder, Mathematische Annalen 98 (1928), 309-312.

[STT] Sleator, D., Tarjan, R., and Thurston, W., Rotation Distance, Triangulations, and Hyperbolic Geometry, Journal of the American Mathematical Society 1 (1988), 647-681.

[Zi] Ziegler, G. M., Lectures on Polytopes, Graduate Texts in Mathematics 152, Springer-Verlag, New York, 1995.

Received August 5, 2000, and in revised form March 29, 2001, and May 3, 2001.

Online publication October 12, 2001. 